\title{
An Effective Approach for Blood Vessel Tree Extraction from Retinal Images with Adaptive Weighted Contrast Adjustment Approach
}

\author{
S. Shafiulla Basha ${ }^{1}$, Dr. K. Venkata Ramanaiah ${ }^{2}$ \\ ${ }^{1,2}$ Department of Electronics and Communication Engineering, Y. S. R Engineering College of Yogi \\ Vemana University, Proddatur, Kadapa-516360, Andhra Pradesh, India
}

\begin{abstract}
Retinal vascular analysis is prominent in diagnosing many diseases like retina diabetes, heart stroke, and retinal damages. Vessel extraction from the retinal images is the crucial part in analyzing and detecting the abnormalities in the retina that predict the nature of the disease. In this paper an effective approach of extracting the blood vessel structures from the retinal images is proposed. The approach is integration of pre processing contrast adjustment process, extraction with segmentation and post processing. The method is tested and evaluated with standard databases which conclude that the proposed approach can be directed to classify and localize the abnormalities. The proposed approach is compared against multiple algorithms and observed that the method could attain an improvement in the accuracy of about $10 \sim 15 \%$. The results obtained in this work reveal that the proposed approach could able to attain an accuracy of $97 \%$.
\end{abstract}

Keywords: Retinal blood vessel, segmentation, adaptive Contrast adjustment, weighted Processing, homogenization

\section{INTRODUCTION}

Retinal blood vessel segmentation and extraction is the key component in analysis and diagnosis of several diseases for ophthalmologists [1]. Among them diabetic retinopathy (DR) is one of the major cause for vision problems. As per clinical reports, diabetes is one among the most challenging chronic health problems. In UK it was estimated that one among twenty persons diagnosed with diabetes and it is predicted that by 2025 around 4 million people may be affected with this disease [2]. According to the estimations of International diabetes federation it was estimated that by 2035 around 592 million people across the globe may be affected with this disease [3]. Timely diagnosis and treatment of this disease may reduce the risk of losing vision. The color fundus photography is an efficient manner of representing the masses of retina in spatial coordinates and to further improve the prediction of disease and abnormality, automatic detection of lesions is developed.

Retinal diagnosis involves several steps like, preprocessing, contrast enhancement, vessel segmentation, extraction, classification, detection and localization. The sequential process steps involved in retinal analysis is depicted in figure 1 . The color fundus retinal image is subjected for contrast adjustment where the intensities are adjusted such that it becomes more convenient to visualize the orientation the blood vessels. Pre-processing focuses on removing the optic disk structure from the image that helps to improve segmentation efficiency. Segmentation and extraction of blood vessel is the key part in the analysis as their orientations and disparities signs the disease severity and its type like diabetes, cardio vascular problems and others [4]. Classification helps to categorize the image there by enables to predict the occurrence of abnormality. Localization and detection is the process involves in finding the abnormalities in the retinal image that is used to diagnose the disease and its severity stage. The work presented in this paper concentrates on contrast adjustment and vessel segmentation.

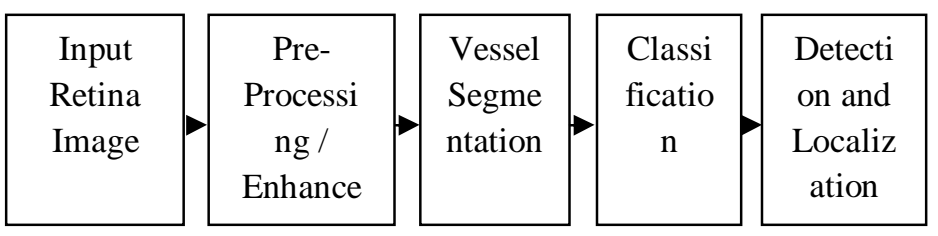

Figure 1: Processes involved in retinal image analysis

The objective of blood vessel segmentation algorithm is to extract the vessels automatically and accurately to analyze the changes in it precisely. With this automation, cost and time involved in grading can be reduced and also the inconsistency that may arise during manual grading can be minimized [5]. The vessel tree segmentation is a challenging and crucial task because of the presence of low contrast and correlated geometrical orientations in the background. The automatic segmentation depends on image quality, application fields; semi automated and 
automated approach and other external factors. Several methods have been proposed so far in the literature for the extraction of vessel tree and most of them yield low sensitivity and accuracy ratio leading to a scope for new methods.

Review of most of the methods can be found in [6], these methods are categorized as supervised and others as semi supervised. The semi-supervised may be unsupervised method finds the patterns of vessel tree without any grouping mechanism. Hunter et.al in [7] suggested a contouring model for vessel border detection. Balas et.al in [8], proposed a histogram based adaptive local thresholding (HALT) approach to extract the useful information for analysis. Zhang and others in [9] extracted a multi dimensional vector with morphological operators and using self organizing map and Ostu thresholding they could mark the outliers of the vessel tree. Oliver et.al in [10] proposed a method for localizing the vessels by summing the responses of Frangi's filter combined with Gabor filter. Bandra et.al in [11] , implemented speeded up adaptive contrast enhancement (SAUCE) approach for enhancing the veins structures and using weighted LAB color model and Isodata the vessel tree structures were extracted. In [1], [12] Kisch's templates were used to extract the vessel structures. This is one of the direct versions of first order derivatives used for detecting the edges. Fraz et. al [13] applied maximum principal curvatures for the extraction of vessel tree from the colored fundus images. The methods presented in [11] [12][13] were compared with respect to the proposed approach .

\section{RETINAL VESSEL TREE SEGMENTATION}

The proposed vessel tree segmentation can be summarized as shown in figure 2

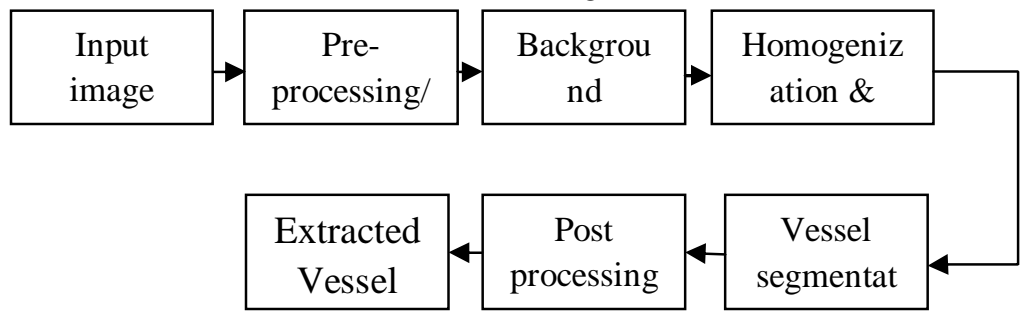

Figure 2: Block diagram of the process involved in vessel extraction
The input images are color fundus images that were imported from standard databases like DRIVE, STARE, CHASE and others

\section{(a) Pre-processing / Contrast Adjustment}

The images that were imported from the databases are usually unprocessed and their intensities may vary unevenly but in order to locate the orientation of blood vessels accurately this images are to be processed. The proposed contrast adjustment algorithm involves conventional CLAHE [14] algorithm with adaptive weighting operation.

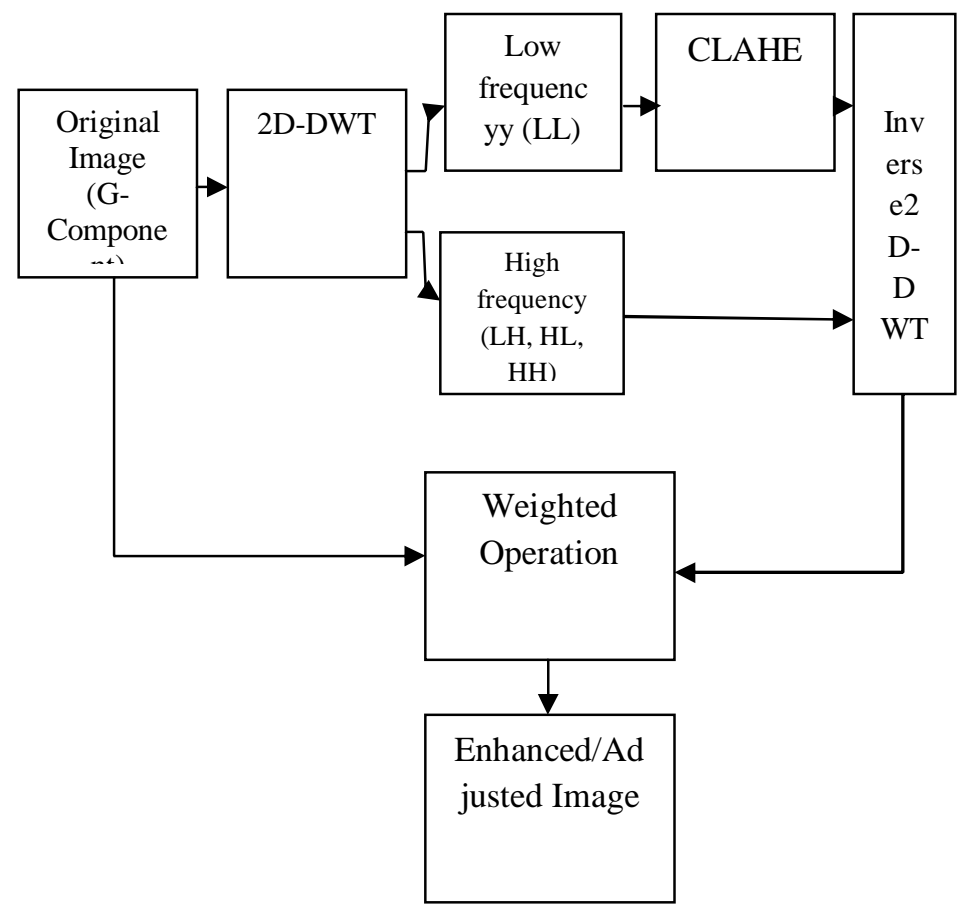

Figure 3: Contrast adjusted process for the retinal fundus image

The blood vessels are usually accompanying a reflex light which has to be minimized. This reflex runs through the central length of the blood vessel and this contrast adjustment process may be termed as central light reflex removal. As in [15], and in most of the retinal pre-processing approaches CLAHE algorithm is used but it often face over enhancement in some portions of the image which is to be treated. The results obtained with proposed approach and conventional CLAHE algorithms were shown in figure 4. 


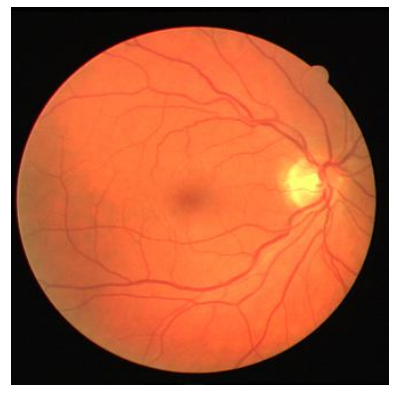

(a)

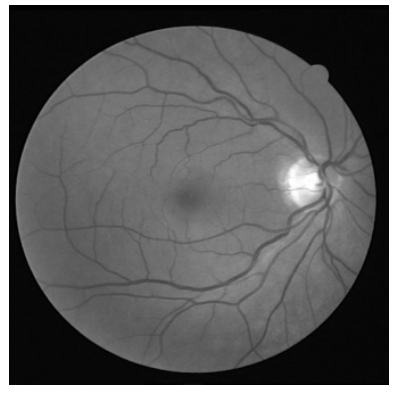

(b)

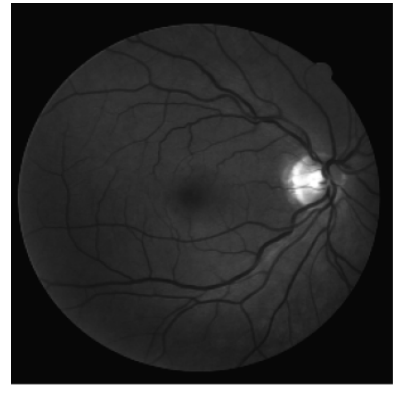

(c)

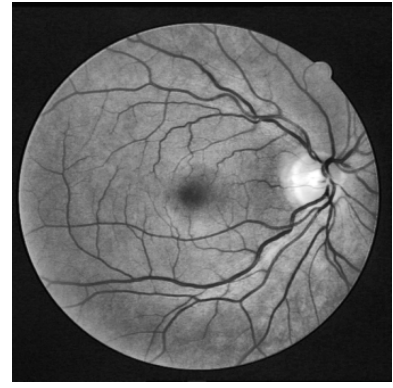

(d)

Figure 4: (a) Original Color fundus Image (b) Green Component (c) Proposed approach (d) CLAHE

The original Green component $\left(\mathrm{I}_{\mathrm{o}}\right)$ image is decomposed into low and high frequency components with 2D discrete wavelet transform and thus obtained low frequency components are applied with CLAHE operation and the high frequency components remains unchanged. The modified low frequency components along with high frequency components are subjected to inverse wavelet operation to obtain a reconstructed image $\left(I_{R}\right)$. An adaptive weighting Operation is performed for the original and reconstructed image to obtain an enhanced image $\left(\mathrm{I}_{\mathrm{E}}\right)$

$$
I_{\bar{E}}=I_{0} * F+\beta ; * I_{R} *(I-F)
$$

Where "I" is the identity matrix and $1<\beta<2$ and "F" can be obtained as $F=f(k)^{\alpha}$ Where $0<\alpha<1$ and $f(k)=\left[k(x, y)^{\alpha}\right]$

It can be observed from the figure 4 that some of the regions in CLAHE are over enhanced but the contrast values are to be limited for accurate segmentation.

\section{(b) Homogenization and vessel Enhancement}

Fundus regions are often corrupted by background intensity variation due to non uniform illumination.
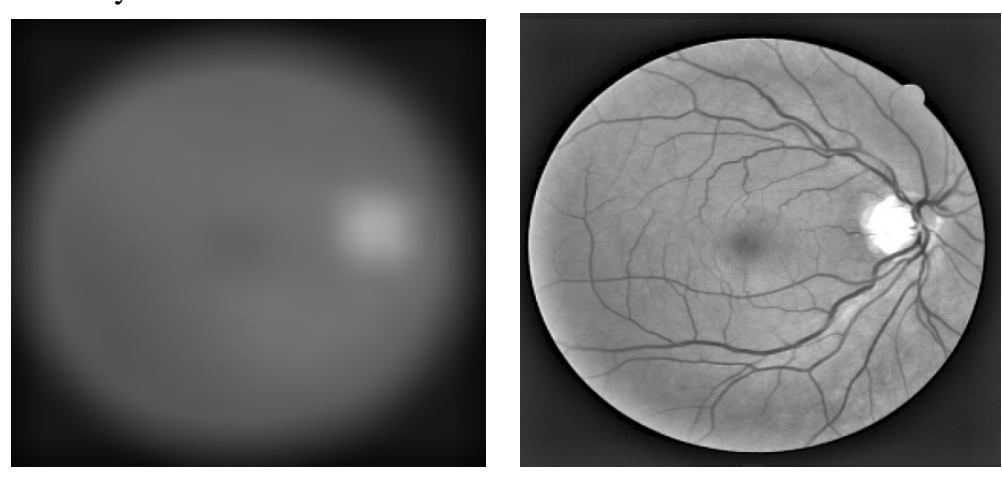

(b)

(a)
On other hand these background pixels may have different intensities in the same image and sometimes these intensities are higher than vessel pixels. This uneven distribution may worsen the performance of the segmentation process hence this has to be mitigated by accurate estimation. This is accomplished by first applying a $3 \times 3$ averaging filter and convolve the resultant image with Gaussian kernel of dimension $9 \times 9$ with zeros mean and variance=1.8. A background image is produced by applying 69x69 mean filters which is further thresholded by following equation

$g_{\text {out }}=\left\{\begin{array}{cl}0 & \text { if } g<0 \\ 255 & \text { if } g>255 \\ g & \text { otherwise }\end{array}\right.$

Where ' $\mathrm{g}$ ' can be obtained by $g=g_{\text {ingut }}+128-g_{\text {max }}$. The final step in this stage is vessel enhancements that is achieved by estimating the complementary of the homogenized image (IH) and apply top hat transformation for it. The resultant images are shown in figure 5. The estimated background image is shown in 5(a), and homogenized image is shown in 5(b) and the vessel enhanced image is shown in 5(c).

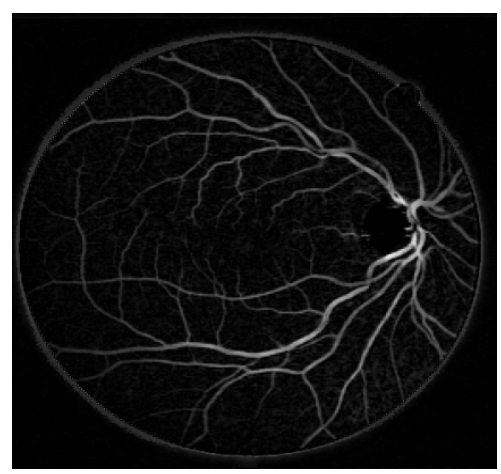

(c)

Figure 5: (a) Estimated Background (b) Homogenized Image (c) Enhanced Vessel Image 
Using the thresholding process and removing the optic disc information from the enhanced image the segmented image can be obtained. When observed the figure 5 the image may contain few gaps and also mis-detected regions that are to be processed. The post processing step involves artifact removal by considering only those regions which have the area more than 5 and classify them as non vessel structures. The outcomes at these stages are shown in figure 6

\section{EXPERIMENTAL RESULTS}

The experiments were conducted using three publicly available databases DRIVE [16], STARE [17], CHASE [18]. The color fundus images are decomposed and the green channel component is used for experiment. The images obtained with these databases are shown in figure 7

In order to quantify and validate the performance of the proposed approach certain metrics were employed where the segmented image is been compared against the manual segmented image from expert.

Table 1: Comparison of manual segmented and automatic segmented

\begin{tabular}{|c|c|c|}
\hline & Vessel Present & Vessel Absent \\
\hline $\begin{array}{l}\text { Vessel } \\
\text { Detected }\end{array}$ & $\begin{array}{ll}\text { True } & \text { Positive } \\
\text { (TP) } & \end{array}$ & $\begin{array}{l}\text { False Positive } \\
\text { (FP) }\end{array}$ \\
\hline $\begin{array}{ll}\text { Vessel } & \text { not } \\
\text { detected } & \end{array}$ & $\begin{array}{l}\text { False Negative } \\
(\text { FN) }\end{array}$ & $\begin{array}{l}\text { True Negative } \\
(\mathrm{TN})\end{array}$ \\
\hline
\end{tabular}

Sensitivity:

$S e=\frac{T F}{T P+F N}$

Specificity: $S p=\frac{T N}{T N+F P}$

Positive Predicted Value: $\quad P F V=\frac{T F}{T F+F F}$

Negative predicted Value: $N P V=\frac{T W}{T N+F N}$

Accuracy: $A c c=\frac{T F+T N}{T F+F P+F N+T N}$

Se, Sp, represent the well classified vessels and non 10 vessels, PPV is the ratio of the pixels that are 11 correctly classified, NPC is the ratio of the pixels of 12 the background that are correctly classified as 12 background; ACC is the global ratio of total correctly 13 classified pixels.
Table 2: Performance analysis of the proposed algorithm with efficiency metrics

\begin{tabular}{cccccc} 
& $\mathbf{S E}$ & $\mathbf{S P}$ & $\mathbf{P P V}$ & $\mathbf{N P V}$ & $\mathbf{A C C}$ \\
1 & 0.763 & 0.987 & 0.845 & 0.978 & 0.9689 \\
2 & 0.7742 & 0.989 & 0.876 & 0.978 & 0.977 \\
3 & 0.1604 & 0.959 & 0.2674 & 0.9246 & 0.891 \\
4 & 0.713 & 0.9895 & 0.8412 & 0.977 & 0.9694 \\
5 & 0.6442 & 0.995 & 0.9088 & 0.9739 & 0.9707 \\
6 & 0.7406 & 0.9943 & 0.9023 & 0.9818 & 0.9775 \\
7 & 0.733 & 0.9788 & 0.722 & 0.9799 & 0.9616 \\
8 & 0.5362 & 0.9925 & 0.7978 & 0.9749 & 0.9687 \\
9 & 0.5909 & 0.9963 & 0.9059 & 0.9755 & 0.9729 \\
10 & 0.7623 & 0.9826 & 0.7531 & 0.9834 & 0.9683 \\
11 & 0.7292 & 0.9811 & 0.7662 & 0.9771 & 0.9614 \\
12 & 0.669 & 0.993 & 0.8901 & 0.9739 & 0.9693 \\
13 & 0.713 & 0.9908 & 0.8562 & 0.9782 & 0.9711 \\
14 & 0.8118 & 0.9863 & 0.8309 & 0.9845 & 0.973 \\
15 & 0.766 & 0.9921 & 0.887 & 0.9814 & 0.9753 \\
\hline AVG & 0.673 & 0.9871 & 0.8031 & 0.9745 & 0.9652
\end{tabular}

\section{COMPARATIVE METHODS}

The proposed algorithm is compared against three methods Coyle filter method with SAUCE in [11], Kirsch's template method [12] and maximum principal curvature method [13]. The pictorial results obtained with the methods are shown in figure 8

Table 3: Segmentation efficiency metrics for SAUCE [11]

\begin{tabular}{lccrc} 
SE & Sp & PPV & \multicolumn{1}{c}{ NPV } & \multicolumn{1}{c}{ ACC } \\
0.6653 & 0.9711 & 0.6747 & 0.9699 & 0.9458 \\
0.6631 & 0.9716 & 0.697 & 0.967 & 0.9439 \\
0.6291 & 0.9695 & 0.6579 & 0.9656 & 0.9405 \\
0.5916 & 0.9753 & 0.6524 & 0.9683 & 0.9475 \\
0.7422 & 0.9682 & 0.6356 & 0.9805 & 0.9524 \\
0.6191 & 0.9739 & 0.6247 & 0.9732 & 0.9501 \\
0.6791 & 0.9708 & 0.6368 & 0.9757 & 0.9503 \\
0.6522 & 0.9782 & 0.6453 & 0.9564 & 0.9452 \\
0.6078 & 0.9728 & 0.5771 & 0.9762 & 0.9518 \\
0.8109 & 0.9595 & 0.5823 & 0.9865 & 0.9499 \\
0.6552 & 0.9723 & 0.6676 & 0.9708 & 0.9476 \\
0.7135 & 0.9656 & 0.6237 & 0.9768 & 0.9469 \\
0.7304 & 0.969 & 0.6431 & 0.9791 & 0.952 \\
0.7239 & 0.9713 & 0.6762 & 0.977 & 0.9524 \\
0.6161 & $0 . .9739$ & 0.655 & 0.9692 & 0.9471
\end{tabular}


$\begin{array}{lllllll}\text { AVG } & 0.6732 & 0.9706 & 0.6432 & 0.9727 & 0.948 & 2\end{array}$

1

2

3

4

5

6

7

8

9

10

11

12

13

14

15

AVG

\begin{tabular}{|c|c|c|c|c|c|}
\hline \multicolumn{5}{|c|}{$\begin{array}{l}\text { Table 4: Segmentation efficiency metrics for Principal curvature } \\
{[13]}\end{array}$} & $\begin{array}{l}1 \\
2\end{array}$ \\
\hline SE & SP & PPV & NPV & $\mathrm{ACC}$ & 3 \\
\hline 0.7742 & 0.9824 & 0.7986 & 0.9797 & 0.9652 & 4 \\
\hline 0.7251 & 0.9905 & 0.8823 & 0.9734 & 0.9667 & 5 \\
\hline 0.8415 & 0.933 & 0.5394 & 0.9844 & 0.9252 & 6 \\
\hline 0.6004 & 0.9958 & 0.9182 & 0.9695 & 0.9671 & 7 \\
\hline 0.7757 & 0.9893 & 0.8445 & 0.9883 & 0.9744 & 8 \\
\hline 0.7329 & 0.9916 & 0.8604 & 0.9812 & 0.9744 & \\
\hline 0.7522 & 0.9732 & 0.6787 & 0.9812 & 0.9577 & \\
\hline 0.8162 & 0.9673 & 0.5784 & 0.9897 & 0.9594 & 10 \\
\hline 0.8203 & 0.9836 & 0.7539 & 0.989 & 0.9742 & 11 \\
\hline 0.844 & 0.9653 & 0.6284 & 0.9889 & 0.9574 & 12 \\
\hline 0.6701 & 0.988 & 0.8254 & 0.9725 & 0.9631 & 13 \\
\hline 0.7609 & 0.9866 & 0.8198 & 0.981 & 0.9699 & 14 \\
\hline 0.7403 & 0.9874 & 0.8174 & 0.9803 & 0.9698 & 15 \\
\hline 0.814 & 0.9844 & 0.8131 & 0.985 & 0.9717 & AVG \\
\hline 0.7107 & 0.9905 & 0.8575 & 0.977 & 0.9696 & \\
\hline 0.7585 & 0.9805 & 0.7744 & 0.9814 & 0.9643 & \\
\hline
\end{tabular}

\begin{tabular}{lllll}
\multicolumn{1}{c}{ SE } & \multicolumn{1}{c}{ SP } & \multicolumn{1}{c}{$\mathbf{P P V}$} & \multicolumn{1}{c}{$\mathbf{~ N P V}$} & \multicolumn{1}{c}{ ACC } \\
0.55 & 0.932 & 0.4347 & 0.958 & 0.903 \\
0.566 & 0.937 & 0.472 & 0.956 & 0.9043 \\
0.346 & 0.951 & 0.397 & 0.939 & 0.899 \\
0.576 & 0.935 & 0.411 & 0.966 & 0.9092 \\
0.487 & 0.952 & 0.433 & 0.9613 & 0.9199 \\
0.492 & 0.948 & 0.407 & 0.9636 & 0.9188 \\
0.582 & 0.9393 & 0.4199 & 0.9676 & 0.9143 \\
0.4764 & 0.9406 & 0.3062 & 0.9073 & 0.9164 \\
0.499 & 0.952 & 0.367 & 0.9659 & 0.9327 \\
0.486 & 0.9502 & 0.404 & 0.9636 & 0.92 \\
0.609 & 0.9344 & 0.4408 & 0.9658 & 0.909 \\
0.45 & 0.9423 & 0.3869 & 0.9562 & 0.9062 \\
0.529 & 0.9391 & 0.399 & 0.9631 & 0.91 \\
0.5881 & 0.9397 & 0.4405 & 0.958 & 0.9134 \\
0.512 & 0.9442 & 0.4318 & 0.956 & 0.9112 \\
0.516 & 0.9423 & 0.4100 & 0.9564 & 0.9124
\end{tabular}

Table 5: Segmentation efficiency metrics for Kirch's template [12]

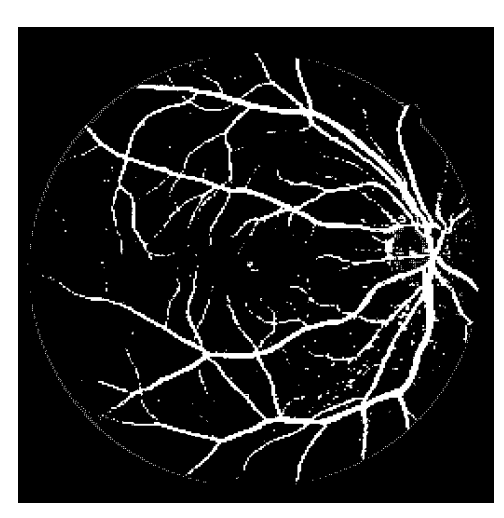

(a)

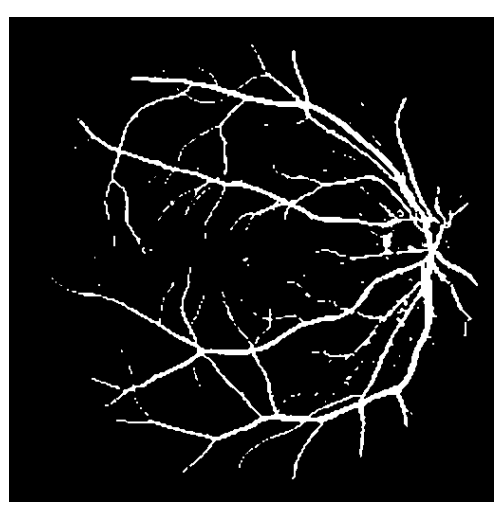

(b)

Figure 6: (a) Segmented Image before post processing (b) After Post processing 


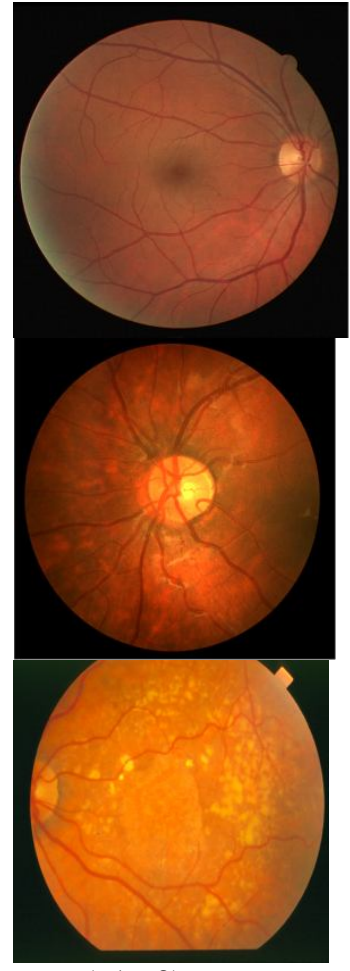

(a1-a3)
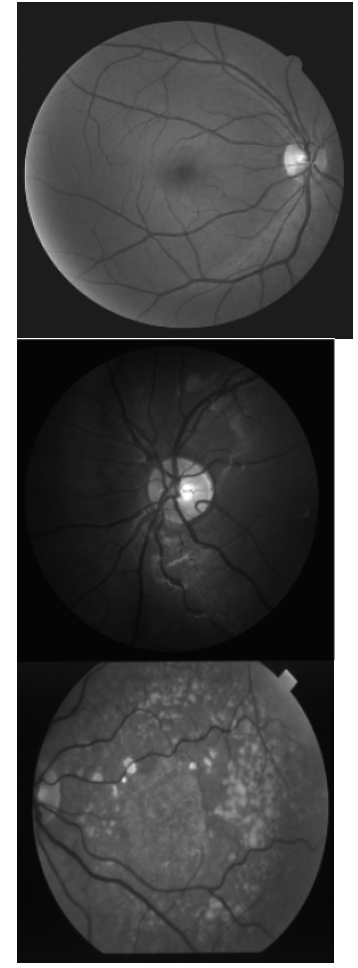

(b1-b3)

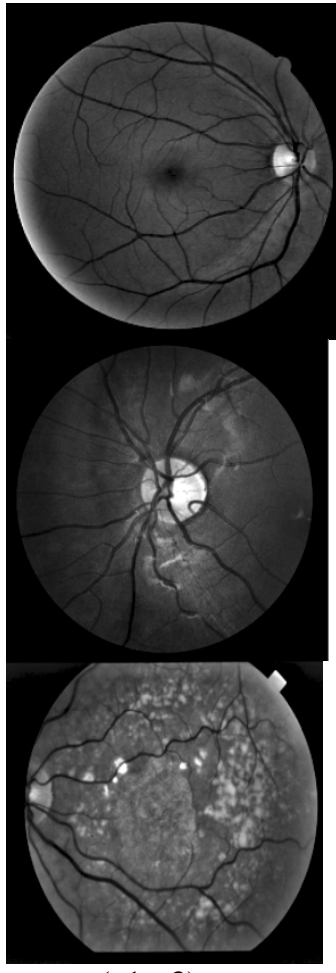

$(\mathrm{c} 1-\mathrm{c} 3)$
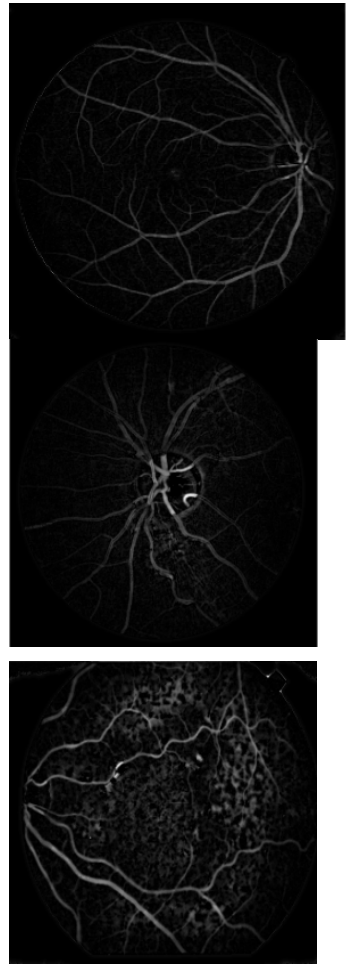

$(\mathrm{d} 1-\mathrm{d} 3)$

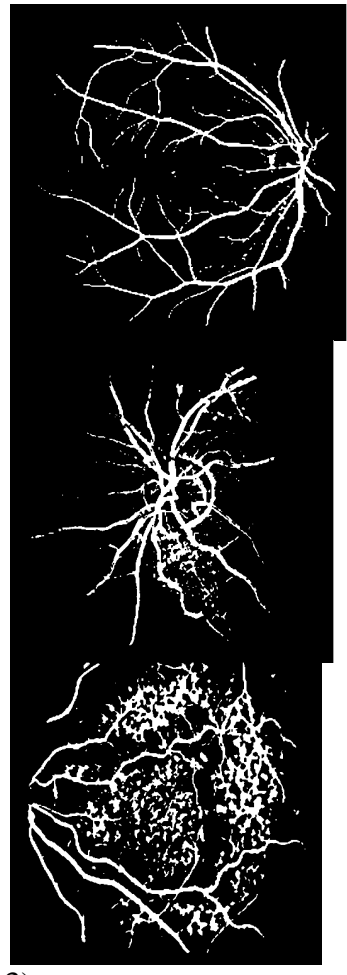

$(\mathrm{e} 1-\mathrm{e} 3)$

Figure 7: First row represents DRIVE dataset, Second row represent CHASE dataset, Third row represent STARE dataset (a1-a3) original Images (b1-b3) Green channel contrast adjusted images (c1-c3) Homogenized images (d1-d3) vessel enhanced images (e1-e3) Extracted vessel tree images.
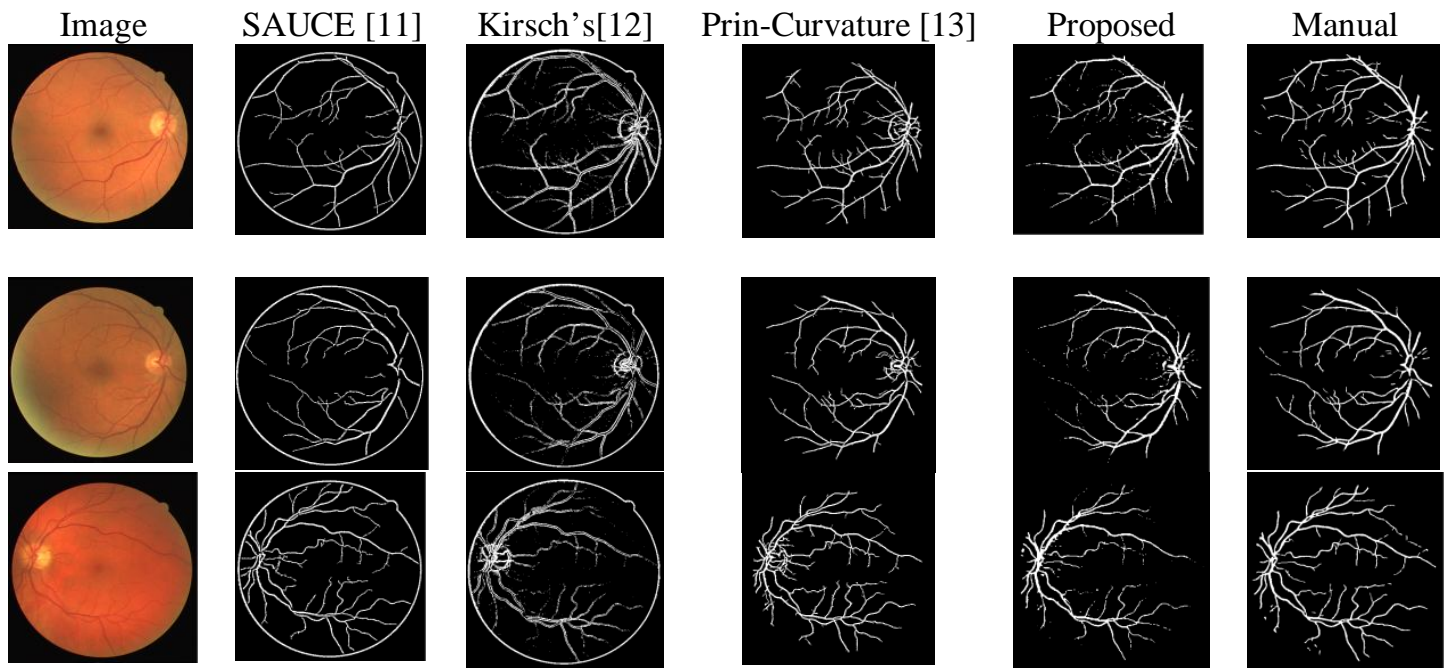

Figure 8: Extracted vessel tree structures with different algorithms, column (1) represents original images from DRIVE dataset, column (2) represents the Coye filter with SAUCE [11], Colum (3) represents Kirch's template based method [12], Column (4) Maximal principal curvature method [13], Column (5) represents the proposed approach, Column (6) represents the manual segmented images with experts. 


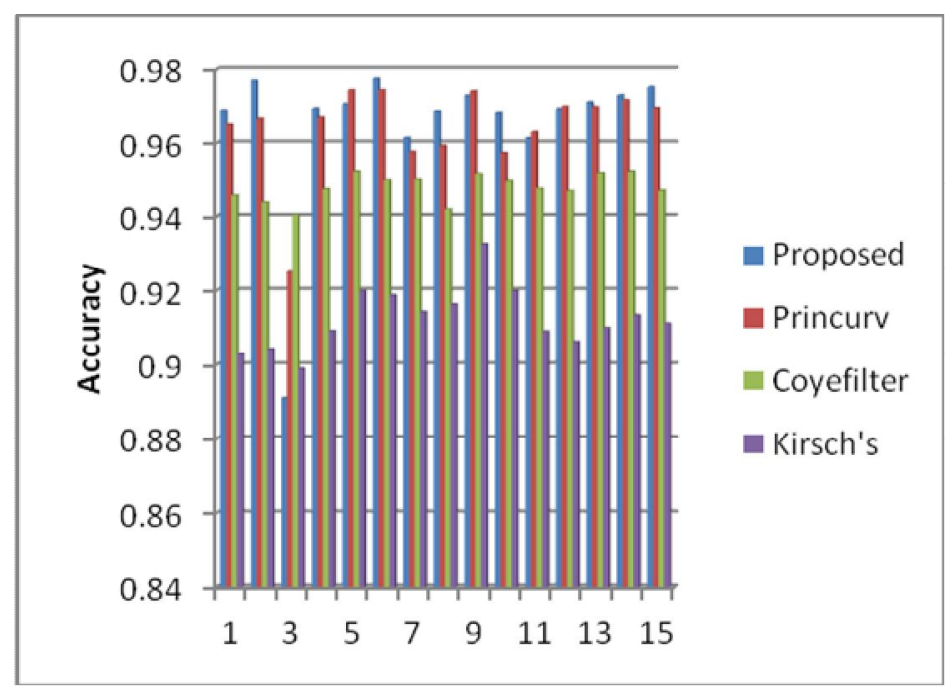

Figure 9: Accuracy assessment of the proposed approach with respect to others

\section{CONCLUSIONS}

An effective contrast adjustment approach with effective vessel extraction is proposed in this paper. The method is evaluated and tested with three standard datasets and compared with three methods in terms of segmentation efficiency metrical analysis. From the results it was observed that the proposed method yields best accuracy than principal curvature around $2.5 \sim 5 \%$, however, it was observed that some

\section{REFERENCES}

[1] Badsha, S., Reza, A.W., Tan, K.G. et al. J Digit Imaging (2013) 26: 1107, https://doi.org/10.1007/s10278-013-9585-8

[2] M.A. Dabbah, J. Graham, I.N. Petropoulos, M. Tavakoli, R.A. Malik, " Automatic analysis of diabetic peripheral neuropathy using multi-scale quantitative morphology of nerve fibres in corneal confocal microscopy imaging, Medical Image Analysis, Volume 15, Issue 5, 2011 https://doi.org/10.1016/j.media.2011.05.016 [3] Guariguata, L., Whiting, D.R., Hambleton, I, 'Global estimates of diabetes prevalence for 2013 and projections for 2035', Diabetes Res. Clin.Pract., 2014, 103, (2), pp. 137-149

https://doi.org/10.1016/j.diabres.2013.11.002 of the vessels are not segmented with principal curvature due to which the PPV value is decreased and this is effectively handled with the current approach. Kirsch's template could able to attain $45 \sim 55 \%$ of PPV which is the limitation of the approach on other hand the current proposed could able to detect around 80 91\% and this figure is almost double of the conventional approach.

[4] Lai, N, "Clinical ophthalmology: a systematic approach", 2004, p. 295

https://doi.org/10.1097/01.opx.0000134900.67908.fc

[5] Pal, A.L, "Extraction of retinal blood vessels from retinal fundus image for computer aided diagnosis", Proc. of National Joint Conf. on Innovations in Engineering \& Technology, 2015, ISBN: 97-8-93-81195-82-6

[6] Fraz, M.M., Remagnin, P, "Hoppe Blood vessel segmentation methodologies in retinal images", Surv. Comput. Methods Programs Biomed., 2012, 108, pp. 407-433 https://doi.org/10.1016/j.cmpb.2012.03.009

[7] Al-Diri, B., Hunter, A., Steel, D.: 'An active contour model for segmenting and measuring 
retinal vessels', IEEE Trans. Med. Imaging, 2009, 28, (9), pp. 1488-1497

https://doi.org/10.1109/TMI.2009.2017941

[8] K. Rapantzikos, M. Zervakis, K. Balas, "Detection and segmentation of drusen deposits on human retina: Potential in the diagnosis of age-related macular degeneration", Medical Image Analysis, Volume 7, Issue 1, 2003

https://doi.org/10.1016/S1361-8415(02)00093-2

[9] Zhang, J., Cui, Y., Jiang, W., et al, "Blood vessel segmentation of retinal images based on neural network", Int. Conf. Image and Graphics, Cham, August, 2015, pp. 11-17

https://doi.org/10.1007/978-3-319-21963-9_2

[10] Oliveira, W.S., Teixeira, J.V., Ren, T.I., et al, "Unsupervised retinal vessel segmentation using combined filters", PLoS ONE, 2016, 11, (2)

https://doi.org/10.1371/journal.pone.0149943

[11] A. M. R. R. Bandara and P. W. G. R. M. P. B. Giragama, "A retinal image enhancement technique for blood vessel segmentation algorithm," 2017 IEEE International Conference on Industrial and Information Systems (ICIIS), Peradeniya, 2017, pp. 1-5.

https://doi.org/10.1109/ICIINFS.2017.8300426

[12] H. Li and O. Chutatape, "Fundus Image Feature Extraction," Proceedings of the 22nd Annual EMBS International conference, Chicago IL, pp. 3071-3073, July 2000

[13] M. M. Fraz, A. Basit, P. Remagnino, A. Hoppe and S. A. Barman, "Retinal vasculature segmentation by morphological curvature, reconstruction and adapted hysteresis thresholding," 2011 7th International Conference on Emerging Technologies, Islamabad, 2011, pp. 1-6 https://doi.org/10.1109/ICET.2011.6048487

[14] Pizer, S.M., Amburn, E.P., Austin, J.D., et al, "Adaptive histogram equalization and its variations", Comput. Vis. Graph. Image Process., 1987, 39, (3), pp. 355-368

https://doi.org/10.1016/S0734-189X(87)80186-X

[15] A. W. Setiawan, T. R. Mengko, O. S. Santoso, and A. B. Suksmono, "Color retinal image enhancement using CLAHE," in ICT for Smart Society (ICISS), 2013 International Conference on, 2013, pp. 1-3.

https://doi.org/10.1109/ICTSS.2013.6588092

[16] https://www.isi.uu.nl/Research/Databases/DRIVE/

[17] http://cecas.clemson.edu/ ahoover/stare/

[18] https://blogs.kingston.ac.uk/retinal/chasedb1/

[19] D. Marin, A. Aquino, M. E. Gegundez-Arias and J. M. Bravo, "A New Supervised Method for Blood Vessel Segmentation in Retinal Images by Using Gray-Level and Moment Invariants-Based
Features," in IEEE Transactions on Medical Imaging, vol. 30, no. 1, pp. 146-158, Jan. 2011.

https://doi.org/10.1109/TMI.2010.2064333 